\title{
Spectroscopic Follow-up of The Unusual Nova OGLE-2015-NOVA-01
}

\section{E. Aydi*1,2}

${ }^{1}$ South African Astronomical Observatory, P.O. Box 9, 7935 Observatory, South Africa

${ }^{2}$ Astronomy Department, University of Cape Town, 7700 Rondebosck, South Africa

E-mail: eaydiasaao.ac.za

The present work addresses spectroscopic follow-up of an unusual nova OGLE-NOVA-2015-01 that was discovered by the Optical Gravitational Lensing Experiment survey. The photometric data obtained for the nova showed a combination of photometric features from different novae classes. With the aim of characterizing it we obtained two spectrocopic observations using the Southern African Large Telescope at the South African Astronomical Observatory. These data showed that it is most probably a classical nova of the Fe II b or Fe II spectroscopic class.

SALT Science Conference 2015 -SSC2015-

1-5 June, 2015

Stellenbosch Institute of Advanced Study, South Africa

\footnotetext{
* Speaker.
} 


\section{Introduction}

Classical novae $(\mathrm{CNe})$ form a subclass of the cataclysmic variable stars. They are "close binary systems consisting of a white dwarf (WD) and a Roche-lobe filling companion" [1]. The WD accretes mass from the Roche-lobe filling companion and once a critical temperature and density are reached, a thermo-nuclear runaway takes place or in other words, a nova eruption [14]. The typical ejecta velocities are $\sim 1000 \mathrm{~km} \mathrm{~s}^{-1}$ and ejecta masses are between $10^{-5}$ and $10^{-4} \mathrm{M}_{\odot}[6,7]$. Several photometric classifications have been used for novae light curves based on the time the nova brightness drops by $2 \mathrm{mag}\left(t_{2}\right)$ or by $3 \mathrm{mag}\left(t_{3}\right)$ [16]. Besides these photometric classifications, spectroscopic classifications were also presented, where the post-outburst spectra are divided into two distinct classes, the Fe II and He/N. The Fe II spectra are characterized by narrow Fe II and HI emission lines. P Cygni profiles can also be present. He/N class spectra are characterized by $\mathrm{He}, \mathrm{N}$ and $\mathrm{HI}$ broad lines. A hybrid class was also introduced showing a combination of the above $[7,9,20]$.

The He/N spectroscopic class are thought to be bright and fast compared to the slower and dimmer Fe II class [5]. The high expansion velocity that characterize the novae of $\mathrm{He} / \mathrm{N}$ class must be the reason for the rapid evolution of this type, however, a precise explanation of the mechanisms responsible for the two different spectroscopic classes is not yet available. The WD mass as well as the circumstellar environment are believed to play an important role in producing the distinct post-outburst spectra [15].

Other eruptions that show many similar characteristics to $\mathrm{CNe}$ are symbiotic novae, which are eruptive cataclysmic variables with a WD and a red giant companion [10] and luminous red novae (LRNe) or infrared luminous red transients (ILRTs) which are stellar explosions thought to be caused by the merger of two stars $[2,18,21]$.

In this work, we present spectroscopic follow-up of OGLE-2015-NOVA-01, which shows an unusual photometric behaviour. The source shows combined features from different novae photometric classes and it presents an interesting case to study. It is important to classify the source prior to establishing the physics behind the eruption characteristics. In Section 2 we summarize the photometric (optical and infra-red) follow-up of the object. SALT (Southern African Large Telescope) spectroscopic optical data [4500 $⿱$ A $-7800 \AA]$ are detailed in Section 3. We present in Section 4 a discussion of the spectroscopic class of the nova, followed by our conclusions in Section 5.

\section{Discovery and photometric data}

OGLE-2015-NOVA-01 was reported on 2015 March 5 by [11] to be a possible nova explosion based on observations from the Optical Gravitational Lensing Experiment (OGLE) survey. The transient was detected by the Early Warning System, which has been designed for the detection of microlensing events. The star is located toward the Galactic bulge at equatorial coordinates $(\alpha, \delta)_{\text {J2000.0 }}=(17: 48: 12.78,-32: 35: 13.44)$. The galactic coordinates of the object are $(l, b)=$ $(15.13,-2.36)$. The nova light curve as well as the OGLE, LCOGT, IRSF and Swift observations and follow-up details can be found on the OGLE website and in $[11,12,13]$.

As a summary of the photometric observations, the object shows a slow light curve with quasiperiodic oscillations during the intensity peak, followed by two faint recoveries after the initial 
decline. These photometric observations show a combination of the different photometric classes ('F' class for flat-topped, 'O' class for oscillations, and 'J' class for jitters) identified by [16]

\section{Spectroscopy data}

\subsection{Observation and data reduction}

Two spectroscopic observations were carried out on 2015 April 22 and May 12, using the Robert Stobie Spectrograph (RSS; [3]; [8]), mounted on the $11 \mathrm{~m}$ SALT situated at the South African Astronomical Observatory (SAAO), Sutherland, South Africa. The observation on 2015 April 22 consisted of two spectral ranges [4500 $⿱-5850 \AA]$ and [5800 $-7000 \AA]$. The RSS long-slit mode was used with a 0.6 " slit at a resolution of $R \sim 5000$ for both spectral ranges with exposure times of $(4 \times 100 \mathrm{~s})$ and $(2 \times 150 \mathrm{~s})$, respectively. The poor weather conditions and seeing on April 22 resulted in a limited S/N. The May 12 observation was carried out under good seeing $(\sim 1.0 ")$. The data consisted of three spectral ranges [4500 $-5450 \AA]$, [5400 -6750 $\AA]$, and [6700 $\AA-7850 \AA$ ]. The RSS long-slit mode was used with the same narrow slit as above resulting in a resolution of $R \sim 7000$ for the first spectral range while a different grating was used for the second and third spectral ranges at a resolution of $R \sim 5000$, with exposure times of $(5 \times$ $200 \mathrm{~s}),(4 \times 100 \mathrm{~s})$, and $(4 \times 100 \mathrm{~s})$, respectively. The spectra were reduced and calibrated using the PySALT pipeline [4]. The images were combined, the backgrounds were subtracted, and the spectra were extracted using the IRAF (Image Reduction and Analysis Facility) software [17].

\subsection{Results}

In Figures 1 to 5, we show the smoothed spectra, where the top spectrum illustrates the April 22 observation and the bottom spectrum represents the May 12 observation. Several broad flat-topped Fe II and H I emission lines appear in the April spectrum. However, the Fe II lines almost disappear in the May spectrum and the $\mathrm{He}, \mathrm{N}$, and high excitation lines appear and become stronger. Both $\mathrm{H} \alpha$ and $\mathrm{H} \beta$ lines show a double-peak in the emission in the May spectrum which may indicate a bipolar morphology of the ejecta. Further in the red, between $6750 \AA$ and $7850 \AA$, the May 12 spectrum shows strong $\mathrm{C}, \mathrm{N}, \mathrm{He}$, and $\mathrm{O}$ emission lines.

The FWHM of $\mathrm{H} \alpha, \mathrm{H} \beta$, and [N II] $5755 \AA$ lines were derived using Lorentzian fitting in order to estimate expansion velocity. We found that for $\mathrm{H} \alpha$ the $\mathrm{FWHM} \sim 2300 \mathrm{~km} \mathrm{~s}^{-1} \pm 200 \mathrm{~km} \mathrm{~s}^{-1}$, for $\mathrm{H} \beta$ the FWHM $\sim 2400 \mathrm{~km} \mathrm{~s}^{-1} \pm 200 \mathrm{~km} \mathrm{~s}^{-1}$, and for [N II] the FWHM $\sim 2700 \mathrm{~km} \mathrm{~s}^{-1} \pm 200$ $\mathrm{km} \mathrm{s}^{-1}$.

\section{Discussion}

The photometric data raises a lot of possibilities including symbiotic nova, LRNe/ILRTs, and $\mathrm{CNe}$. The most likely possibility is a $\mathrm{CNe}$ showing some unusual behaviour. The aim of this work is to identify the spectroscopic class of the nova from the SALT data. As mentioned previously, $\mathrm{CNe}$ are divided into two spectroscopic classes (Fe II and $\mathrm{He} / \mathrm{N}$ ).

Concerning this object, the April spectrum is dominated by Fe II and HI lines which are characteristic of the Fe II spectroscopic class. The lines are moderately broad and show a flat-top which 


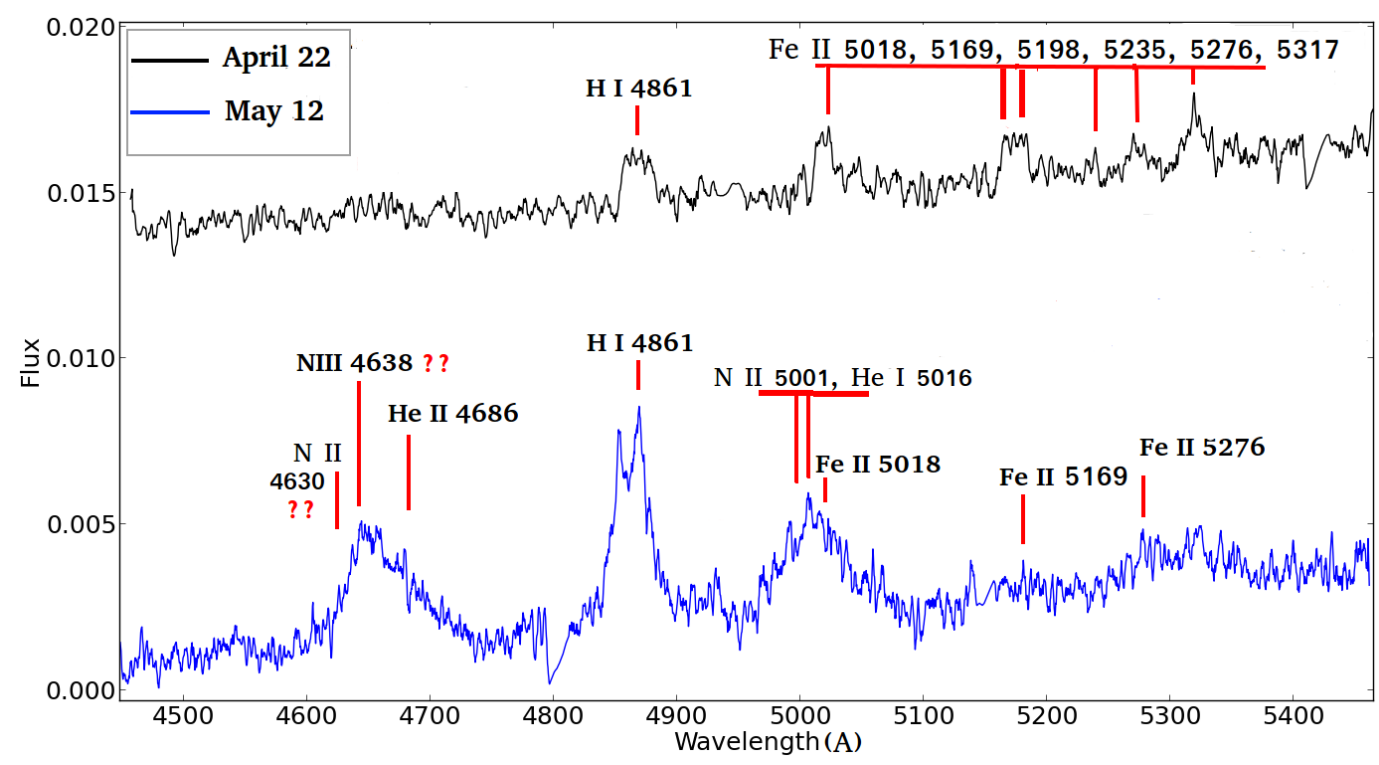

Figure 1: The flux plotted to arbitrary units between $4440 \AA$ and $5450 \AA$. The April 22 spectrum (upper) shows several broad Fe II emission lines, weak He II and $\mathrm{N}$ III emission lines, and $\mathrm{H} \beta$ line. However, in the May 12 spectrum (lower), the Fe II lines become weaker and almost disappear in contrast to the $\mathrm{He}$ and $\mathrm{N}$ lines that become stronger. The $\mathrm{H} \beta$ line shows a double-peak. For clarity, the April 22 spectrum is vertically shifted. In the April 22 spectrum, the chip gaps are between: 4927.2 $\AA$ and $4953.4 \AA$ and between: $5409.0 \AA$ and $5433.9 \AA$. In the May 12 spectrum, the chip gaps are between: $4790.9 \AA$ and $4810.0 \AA$ and between: $5140.5 \AA$ and $5158.4 \AA$.

opens the possibility of a Fe II b class which is a hybrid class showing a transition from Fe II to $\mathrm{He} / \mathrm{N}$ class with broad lines [19]. In the May spectrum, the Fe II lines almost disappear while He, $\mathrm{N}$, and $\mathrm{O}$ lines indicating a He/N spectral type which increase the possibility of a hybrid Fe II $\mathrm{b}$ spectral type. One of the features that distinguishes the Fe II from the $\mathrm{He} / \mathrm{N}$ is the expansion velocity; Fe II class have an expansion velocity less than $\sim 2500 \mathrm{~km} \mathrm{~s}^{-1}$, while $\mathrm{He} / \mathrm{N}$ class have an expansion velocity greater than $\sim 2500 \mathrm{~km} \mathrm{~s}^{-1}$. The expansion velocity of this object is $\sim 2500$ $\mathrm{km} \mathrm{s}^{-1}$, i.e. both classes (Fe II and Fe II b) are possible. The flat-topped lines in the April spectrum are also one of the characteristics of Fe II b class.

As a conclusion, our target shows a possible hybrid Fe II b or a Fe II spectroscopic class in a transition to the nebular phase with a slow light curve peak combining different light curves classes (' $\mathrm{F}$ ', ' $\mathrm{J}$ ', and ' $\mathrm{O}$ '). This combination of different novae features that are rarely observed for one object poses new question about the novae classification and eruption mechanisms and makes the present object an interesting case study.

\section{Conclusions}

Besides that the object shows a nova spectral behavior, based on the available spectroscopy, no clear spectral classification of the object is reached, however, both possibilities of Fe II b or Fe II 


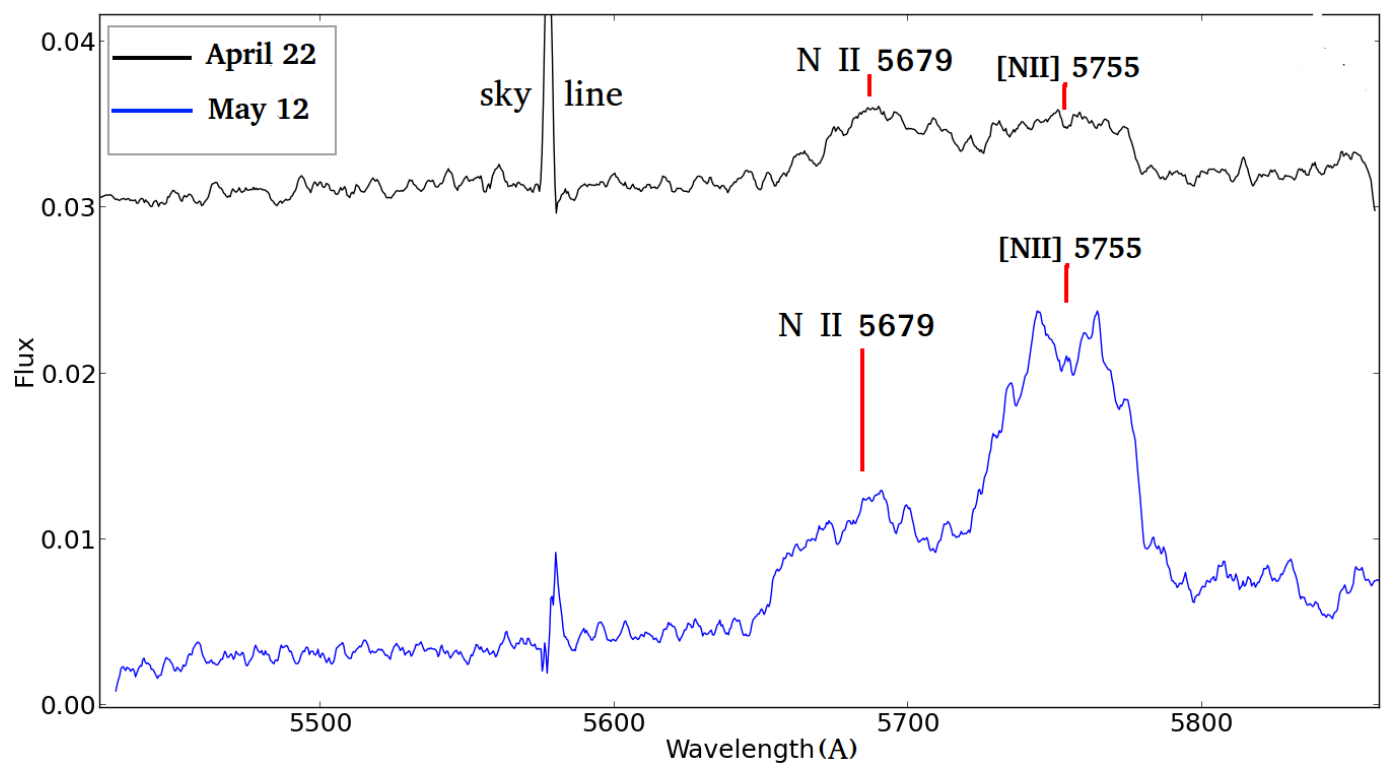

Figure 2: The flux plotted to arbitrary units between $5400 \AA$ and $5850 \AA$. The April 22 spectrum (upper) shows broad and relatively weak N II $5686 \AA$ line and [N II] $5755 \AA$ line. In the May 12 spectrum (lower), the [N II] line becomes stronger compared to the N II line. For clarity, the April 22 spectrum is vertically shifted.

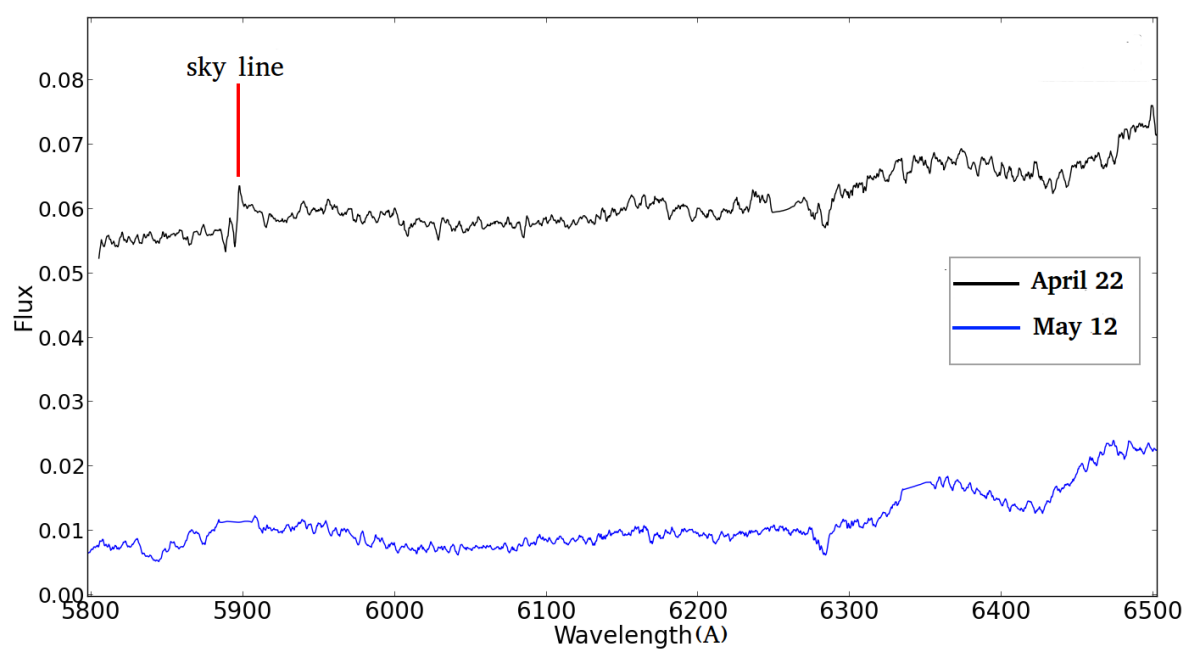

Figure 3: The flux plotted to arbitrary units between $4440 \AA$ and $5450 \AA$ between $5850 \AA$ and 6500 $\AA$. No strong emission lines are present in this spectral range. For clarity, the April 22 spectrum is vertically shifted. In the April 22 spectrum, the chip gap is between: $6242.8 \AA$ and $6267.1 \AA$. In the May 12 spectrum, the chip gaps are between: $5877.9 \AA$ and $5902.7 \AA$ and between: 6332.6 $\AA$ and $6355.9 \AA$. 

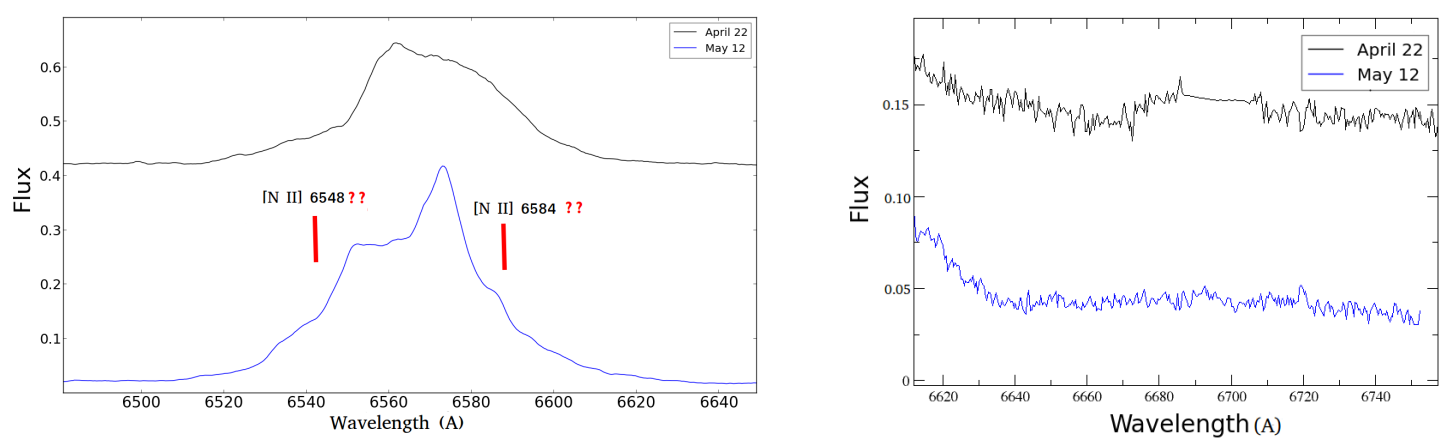

Figure 4: The flux plotted to arbitrary units between (a) $6480 \AA$ and $6640 \AA$ (b) from $6620 \AA$ and $6750 \AA$. The April 22 spectrum (upper) shows broad $\mathrm{H} \alpha$ emission line (FWHM $\sim 2300 \mathrm{~km} \mathrm{~s}^{-1} \pm$ $200 \mathrm{~km} \mathrm{~s}^{-1}$. In the May 12 spectrum (lower), the $\mathrm{H} \alpha$ line shows a double-peak similar to the one of $\mathrm{H} \beta$. Both [N II] $6548 \AA$ and [N II] $6584 \AA$ are expected to be present but merged with the broad $\mathrm{H} \alpha$. For clarity, the April 22 spectrum is vertically shifted. In the April 22 spectrum, the chip gap is between: $6685.5 \AA$ and $6708.1 \AA$.

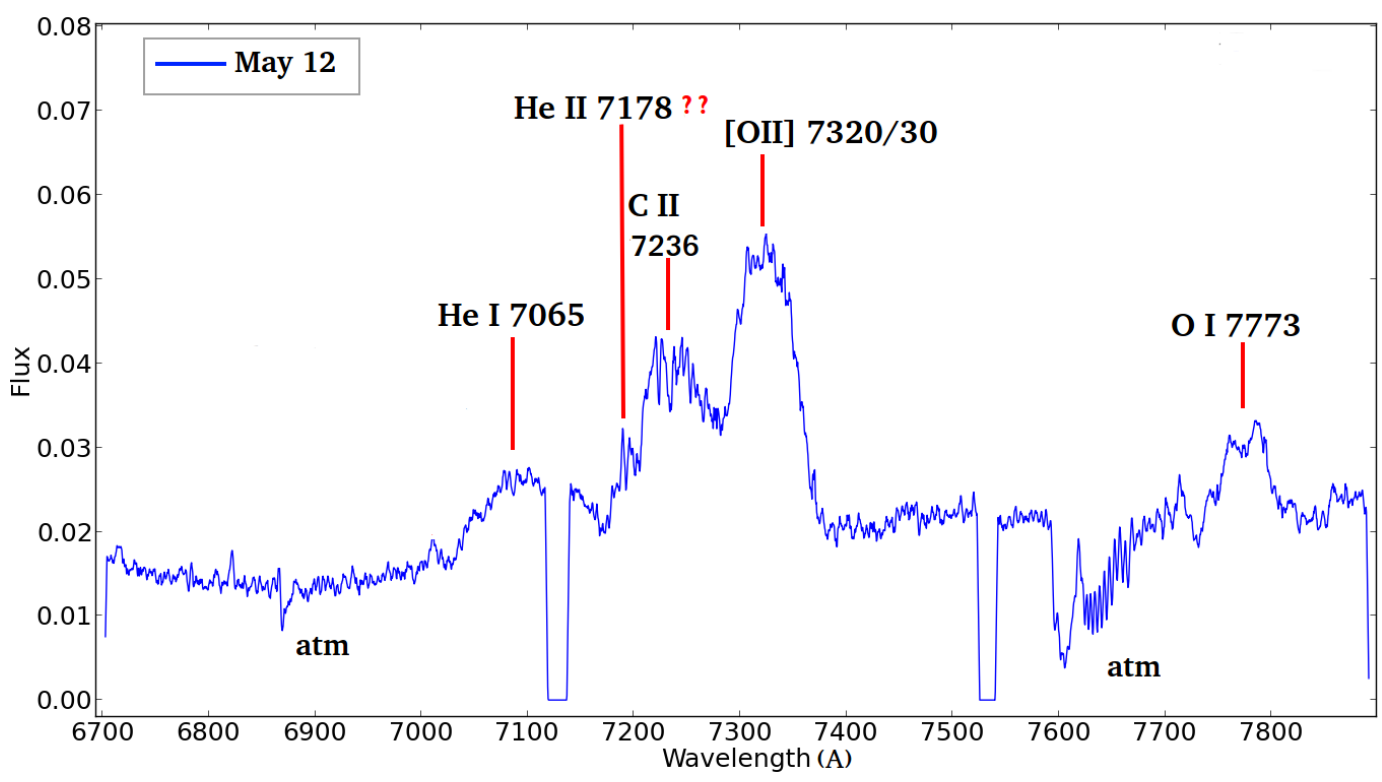

Figure 5: The flux plotted to arbitrary units between $6750 \AA$ and $7850 \AA$. The May 12 spectrum shows strong $\mathrm{C}, \mathrm{N}$ and $\mathrm{O}$ emission lines. In the May 12 spectrum, the chip gaps are between: 7111.8 $\AA$ and 7134.4 $\AA$ and between: $7521.4 \AA$ and $7542.1 \AA$. 
classes are present. Combined with the photometric behaviour, the nature of OGLE-2015-NOVA01 is best described as an unusual one. Based on the available data, the most likely possibility is a slow FeII or Fe II b nova in transition with a combination of different light curve classes ('F', 'J', and 'O'). Tracking the detailed spectroscopic and photometric evolution of the object is essential for a definitive classification. A detailed study of the elemental abundances is also essential. Hence, further observations are needed to certainly identify the object and understand the physical mechanism responsible for this explosion.

\section{Acknowledgments}

A part of this work is based on observations made with the Southern African Large Telescope (SALT), under the program 2014-2-DDT-006, and the IRSF, and makes use of observations from the LCOGT network and OGLE data. The IRSF project is a collaboration between Nagoya University and the South African Astronomical Observatory (SAAO) supported by the Grants-in-Aid for Scientific Research on Priority Areas (A) (No. 10147207 and No. 10147214) and Optical \& Near-Infrared Astronomy Inter-University Cooperation Program, from the Ministry of Education, Culture, Sports, Science and Technology (MEXT) of Japan and the National Research Foundation (NRF) of South Africa. EA gratefully acknowledge the receipt of research grants from the National Research Foundation (NRF) of South Africa. We are grateful to Steve Crawford, Marissa Kotze, Brent Miszalski, and Petri Vaisanen for assistance with the SALT observations and data reductions. This work makes use of observations from the LCOGT network, which includes three SUPAscopes owned by the University of St Andrews. The Gaia transient follow-up program uses equal network time allocations from the University of St Andrews and the South African Astronomical Observatory (SAAO).

We acknowledge helpful discussions with Joanna Mikołajewska, Krystian Iłkiewicz, and Katarzyna Drozd.

\section{References}

[1] M. F. Bode \& A. Evans, TClassical Novae, Cambridge University Press, Cambridge 2008.

[2] H. E. Bond et al., The 2008 Luminous Optical Transient in the Nearby Galaxy NGC 300, ApJ 695 (2009) L154

[3] E. B. Burgh et al., Instrument Design and Performance for Optical/Infrared Ground-based Telescopes, in proceedings of Photo-Optical Instrumentation Engineers (SPIE) Conference Series, Vol. 4841, pages 1463-1471 (2003)

[4] S. M. Crawford, Society of Photo-Optical Instrumentation Engineers (SPIE) Conference Series, in proceedings of In Society of Photo-Optical Instrumentation Engineers (SPIE) Conference Series, Vol. 7737, page 25 (2010)

[5] M. Della Valle \& M. Livio, The Spectroscopic Differences between Disk and ThickDisk/Bulge Novae, ApJ 506 (1998) 818 
[6] J. S. Gallagher \& S. Starrfield, Theory and observations of classical novae, ARA\&A 16 (1978) 171

[7] C. H. P. Gaposchkin, The galactic novae., Interscience Publishers, New York 1957

[8] H. A. Kobulnicky, Instrument Design and Performance for Optical/Infrared Ground-based Telescopes, in proceedings of In Society of Photo-Optical Instrumentation Engineers (SPIE) Conference Series, Vol. 4841, pages 1463-1471 (2003)

[9] D. B. Mclaughlin, The spectral changes of a typical nova, Popular Astronomy 52 (1944) 109

[10] J. Mikolajewska \& S. J. Kenyon, On the nova-like eruptions of symbiotic binaries, MNRAS 256 (1992) 177

[11] P. Mroz \& A. Udalski, OGLE-2015-NOVA-01: A possible classical nova?, The Astronomer's Telegram 7179 (2015) 1

[12] P. Mroz et al., OGLE-2015-NOVA-01 is getting brighter, The Astronomer's Telegram 7394 (2015) 1

[13] P. Mroz et al., Spectroscopic and photometric follow-up of OGLE-2015-NOVA-01, The Astronomer's Telegram 7472 (2015) 1

[14] D. Prialnik, The evolution of a classical nova model through a complete cycle, ApJ 310 (1986) 222

[15] A. W. Shafter et al., A Spectroscopic and Photometric Survey of Novae in M31, ApJ 734 (2011) 12

[16] R. J. Strope et al., Catalog of 93 Nova Light Curves: Classification and Properties, AJ 140 (2010) 34

[17] D. Tody, Instrumentation in astronomy VI, in proceedings of in Society of Photo-Optical Instrumentation Engineers (SPIE)Conference Series, Vol. 627, page 733 (1986)

[18] R. Tylenda et al., V1309 Scorpii: merger of a contact binary, A\&A 528 (2011) A114

[19] R. Williams, Origin of the "He/N" and "Fe I" Spectral Classes of Novae, AJ 144 (2012) 98

[20] R. E. Williams, The formation of novae spectra, AJ 104 (1992) 725

[21] S. C. Williams et al., A Luminous Red Nova in M31 and Its Progenitor System, ApJ 805 (2015) L108 